\title{
HELICHRYSUM ITALICUM ESSENTIAL OIL PREVENTS SKIN LIPIDS PEROXIDATION CAUSED BY POLLUTION AND UV
}

\author{
Combes', M Legrix', Rouquet', SRivoire', S Grasset', Cenizo $^{1}$, A Moga ${ }^{2}$ and P Portes'
}

\section{INTRODUCTION}

The increase in air pollution over the years has had major effects on human skin. Clinical studies conducted in cities having high pollution levels demonstrated significantly reduced vitamin $\mathrm{E}$ and squalene levels, higher sebum and carbonylated proteins levels. Ozone $\left(\mathrm{O}_{3}\right)$ and air pollutants exposure contribute to the generation of reactive oxygen species (ROS) that interact with skin surface lipids and initiate the lipid peroxidation reaction cascade. This oxidative stress has been suggested to eventually overwhelm the skin's natural defenses depleting its antioxidant capacity, such as vitamins $C$ and $E$, and disrupting the skin barrier function. Once skin defenses are overcome, oxidation of squalene, representing $\sim 10 \%-15 \%$ of sebum, gives rise to reactive by-products penetrating deeper into skin layers. UV exposure and $\mathrm{O}_{3}$ have also been found to have an additive effect on antioxidant vitamin $\mathrm{E}$ (Vit E) depletion and lipid peroxidation levels.

For people living in urban areas, chronic exposure to UV and air pollutants contribute to create a background inflammatory environment that in turn leads to acne and premature skin aging.

The aim of this study was to evaluate the antioxidant and protective properties of Helichrysum italicum essential oil (HIEO) in vitro and in vivo when used in a cosmetic formula.

\section{MATERIAL AND METHODS}

Oxygen Radical Absorbance Capacity (ORAC) Assay. The method used was described by Cao et al. 1993. Peroxyl radical was generated using 2,2'-azobis (2 amidinopropane) dihydrochloride (AAPH) and fluorescein was used as probe. Fluorescence decay was monitored kinetically at $37^{\circ} \mathrm{C}$. Trolox was used as antioxidant reference and HIEO values are expressed as $\mu$ mol Trolox equivalent.

Conjugated Dienes Method. In this assay, liposomes mimicked cell membranes. After 5 h at $45^{\circ} \mathrm{C}$, conjugated dienes generated by lipids oxidation were followed by reading absorbance at $233 \mathrm{~nm}$. A lower amount of conjugated dienes compared to the control reflects liposomes protection.

Squalene protection assay. Squalene was incubated with pollutants (Singlet oxygen or Ozone) to induce its degradation. During the stress, squalene was incubated with Vit $\mathrm{E}$ (a-tocopherol acetate), the antioxidant reference, HIEO or a formula containing HIEO + Vit E. Protected squalene was measured by GC/MS.

Clinical Evaluation. 51 volunteers, mean age 56 years, living in urban area (Marseille, France) applied the formula twice a day onto the face for 2 months. Sebum on the forehead was collected and high resolution digita photographs were taken on Day 0 (D0), D28 and D56. Squalene monoperoxide was measured by LC/MS method and Vit $E$ by $\mathrm{LC} /$ Fluo method. Analysis of the brightness of the skin and wrinkles appearance was carried out with the software FrameScan 3.3 (OrionTechnoLab ${ }^{T M}$ ).

\begin{tabular}{|c|c|c|}
\hline \multicolumn{3}{|c|}{$\begin{array}{l}\text { HIEO is rich in antioxidant and } \\
\text { anti-inflammatory compounds: }\end{array}$} \\
\hline COMPOUND & $\%$ & PROPERTIES \\
\hline Neryl acetate & 32.8 & 1 \\
\hline$\gamma$-curcumene & 12.31 & $A-0 x$ \\
\hline Italidiones 1 and 2 & 10.34 & A-Inf \\
\hline Limonene & 4.83 & $A-0 x / A-\ln f$ \\
\hline Neryl propionate & 4.66 & 1 \\
\hline Linalol + italicenes & 4.29 & A-Inf \\
\hline$\beta$-eudesmol & 3.57 & 1 \\
\hline a-curcumene & 2.59 & $A-0 x$ \\
\hline a-pinene & 2.02 & 1 \\
\hline Nerol & 2.01 & A-Inf \\
\hline$\beta$-caryophyllene & 1.57 & $A-0 x$ \\
\hline 1.8-cineole (eucalyptol) & 0.82 & A-Inf \\
\hline$\beta$-pinene & 0.68 & 1 \\
\hline a-cedrene & 0.63 & 1 \\
\hline
\end{tabular}

HIEO is rich in antioxidant (A-0x) and anti-inflammatory (A-inf) molecules.

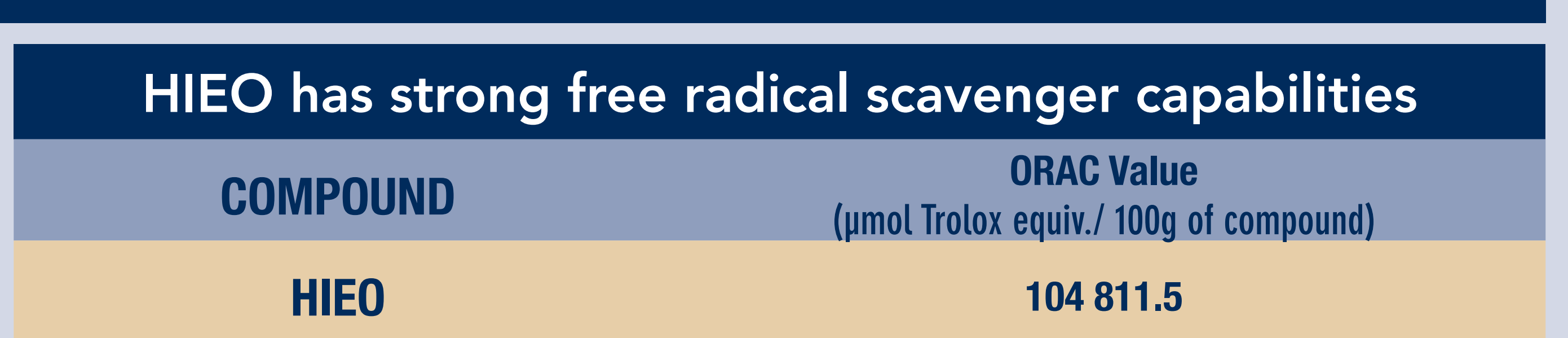

HIEO has a high ORAC value showing a good antioxidant activity. This value is similar to that obtained for acai berries (102 700), one of the best food antioxidants (USDA Database for the ORAC of Selected Foods. 2010)

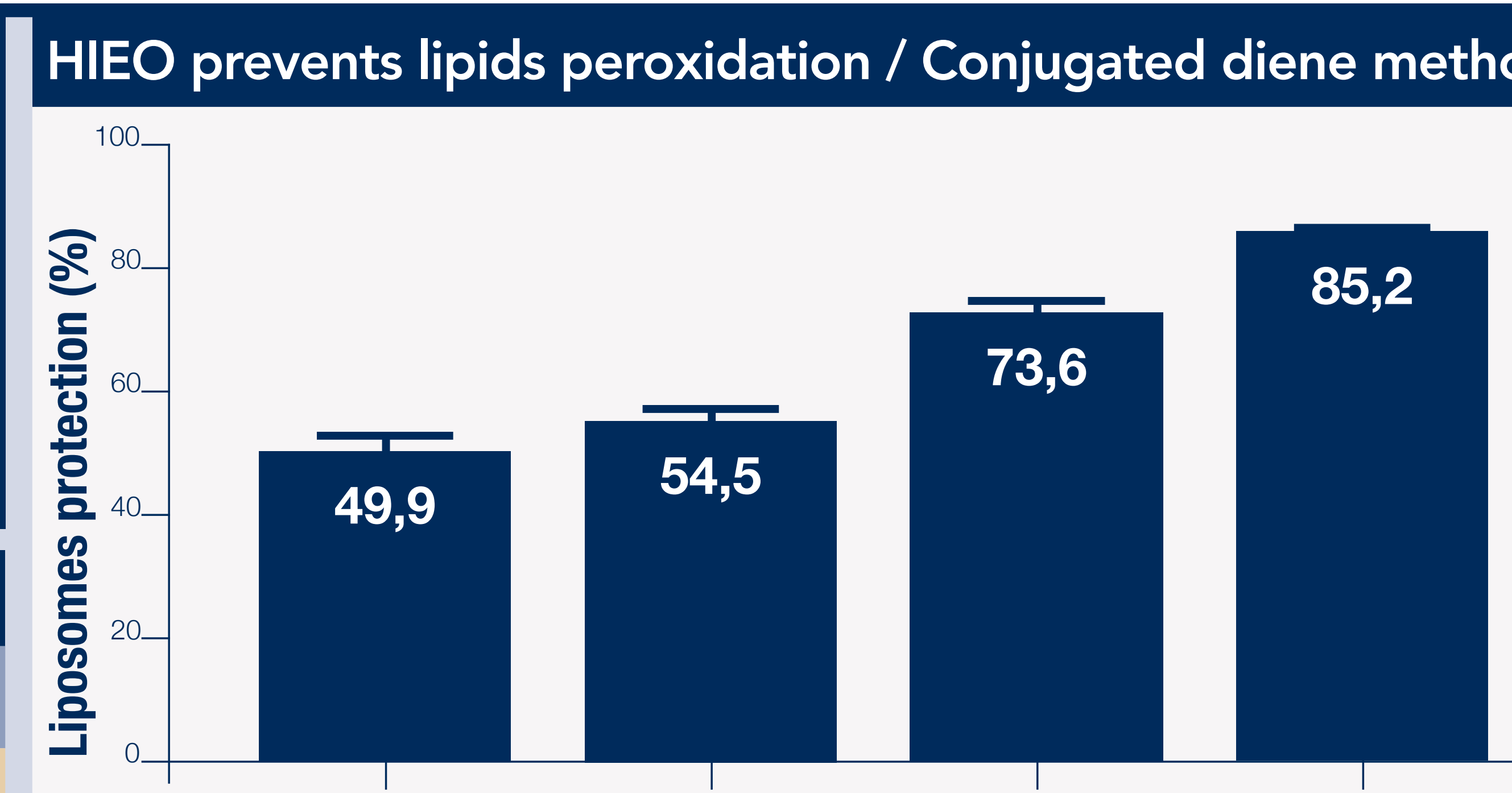

HIEO concentration \%

HIEO protected liposomes from peroxidation in a dose-effect manner with up to $85,2 \%$ protection when used at $0,3 \%$
HIEO Protects Squalene from degradation by pollutants

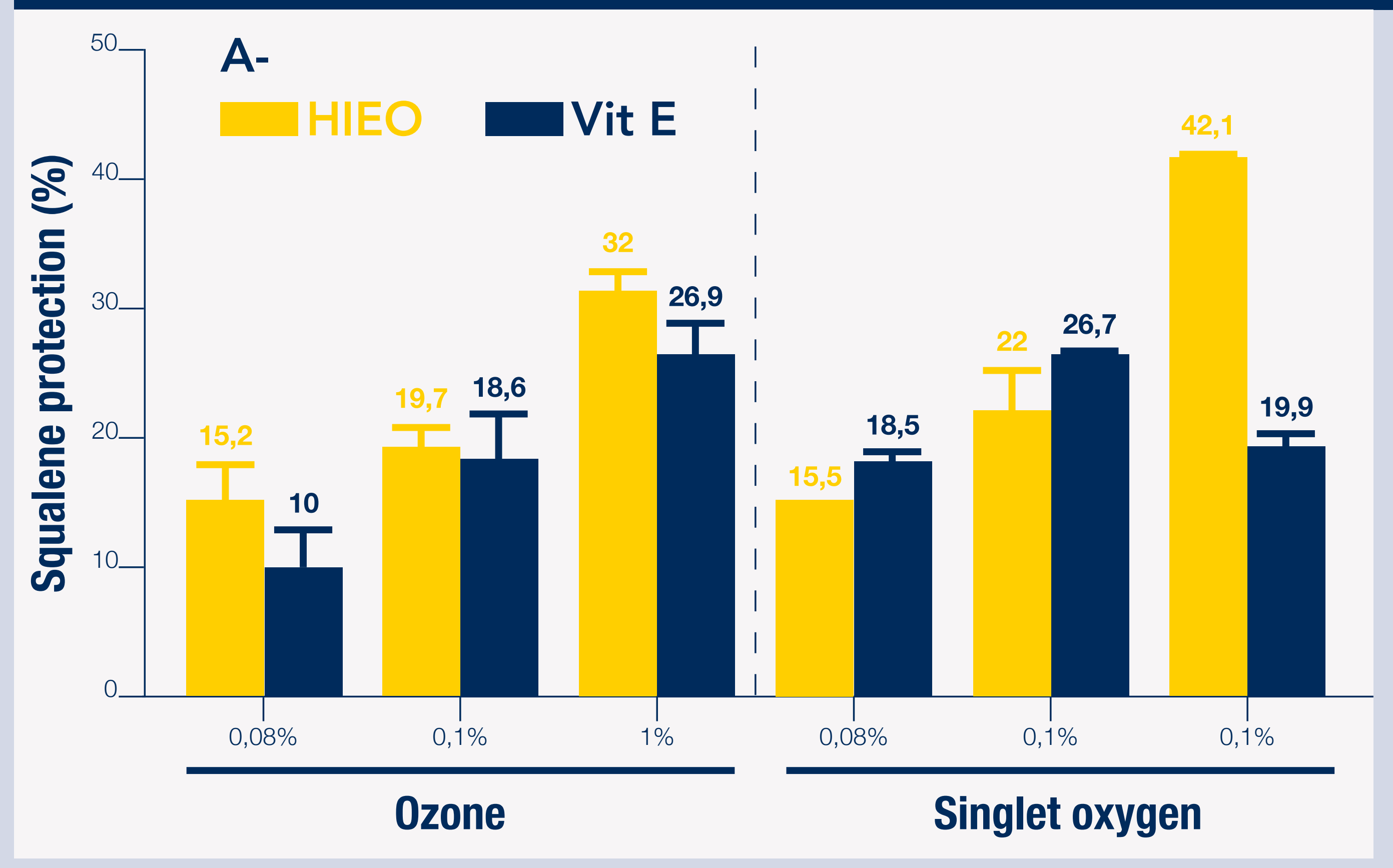

A- Using squalene as a simplified model of sebum, $0.1 \%$ and $1 \%$ HIEO better prevented squalene oxidation under ozone than Vit $E$ at the same concentrations. Under singlet oxygen exposure, while HIEO and Vit $\mathrm{E}$ values were comparable at $0.1 \%$, only HIEO efficacy increased at higher concentration $(42.1 \%$ at $1 \%)$.

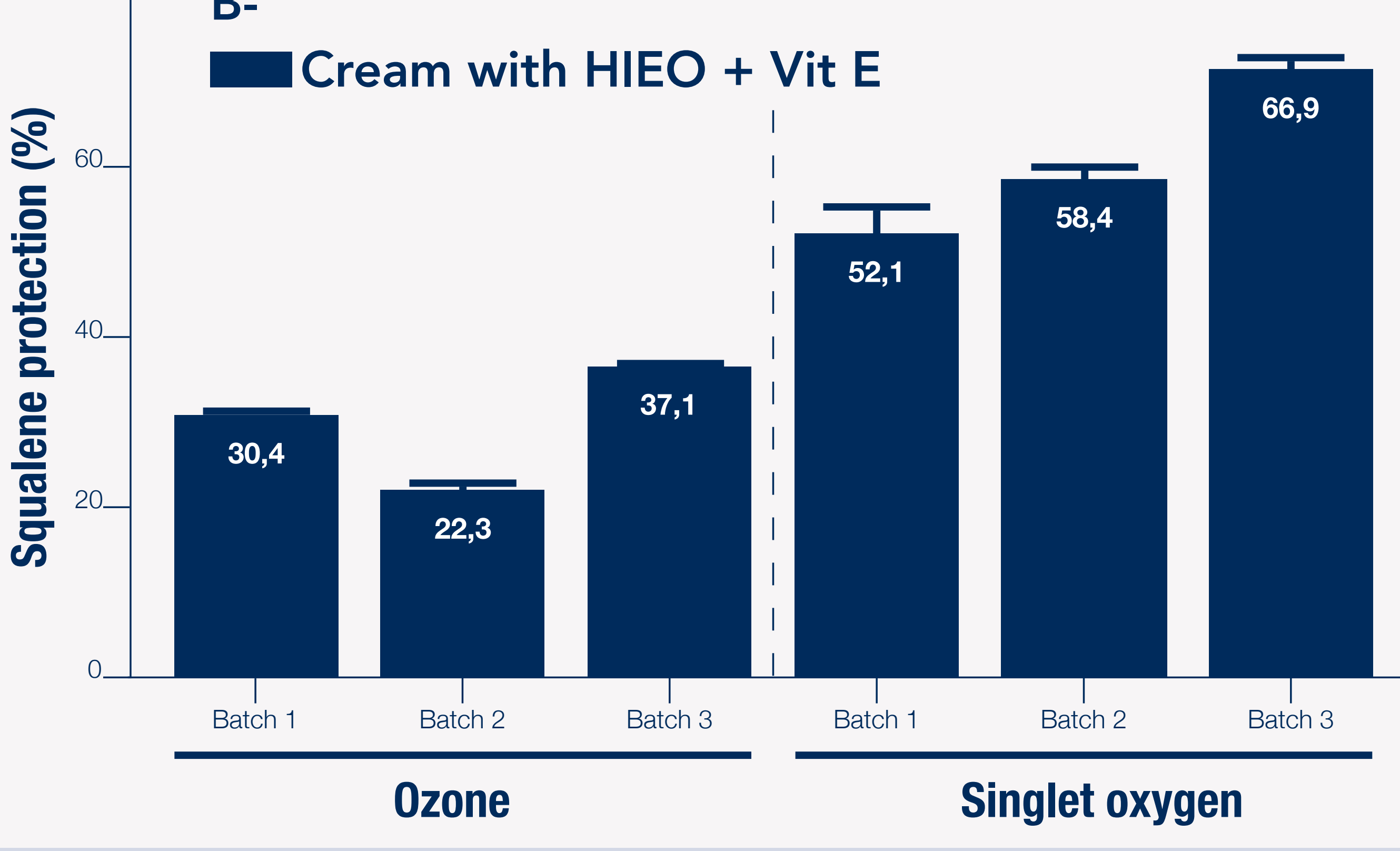

B- A cosmetic formula containing HIEO and Vit E showed even higher protection under Ozone and singlet oxygen exposure suggesting a synergy between the two compounds. Clinical Evaluation in urban area

Clinical Evaluation in urban area
C- Wrinkles and Brightness reduction

\section{CONCLUSION}

Helichrysum italicum essential oil has an antioxidant and anti-inflammatory-rich composition. In vitro, HIEO protected lipids and in particular squalene from peroxidation and degradation with better efficacy than vitamin $E$ at $1 \%$. A formula containing HIEO and vitamin E showed even better results. In a clinical study in urban area, the formula improved sebum composition and skin quality suggesting that skin's natural defenses have been restored.

Taken together, these results suggest that Helichrysum italicum essential oil could protect skin from pollution and UV induced oxidative stress. Its radical scavenger properties seem complementary to that of Vitamin E. They could act synergistically to better protect skin than increasing Vit $\mathrm{E}$ concentration alone.

\section{L'OCCITANE}

E N R O E N C E 